\title{
ANÁLISIS BIBLIOMÉTRICO SOBRE LA PSICOPATOLOGÍA Y PSICOLOGÍA CLÍNICA EN EL ÁMBITO MILITAR
}

\author{
ANA MAGAZ, BONIFACIO SANDÍN, PALOMA CHOROT Y MIGUEL A. SANTED
}

\author{
Universidad Nacional de Educación a Distancia
}

(Aceptado en marzo de 2004)

\begin{abstract}
El objetivo del presente trabajo ha consistido en llevar a cabo un análisis bibliométrico basado en una revisión cuantitativa sobre la investigación en psicopatología y psicología clínica relacionada con el ámbito militar, producida entre los años 1992 y 2002 y referenciada en la base de datos PsycINFO. Se analizaron un total de 526 estudios publicados durante este periodo de 11 años. El país más productivo, con gran diferencia, fue Estados Unidos, seguido de China y Reino Unido. Military Medicine fue con mucho la revista que publicó el mayor número de estudios, seguida de Psychiatric Services, Journal of Clinical Psychology, y Military Psychology. Casi un tercio de las publicaciones se referían a estudios de prevención o tratamiento.
\end{abstract}

Palabras clave: Psicología militar, psicopatología, psicología clínica, análisis bibliométrico.

\section{Publication trends in military psychopathology and clinical psychology}

The aim of this work was to conduct a bibliometric quantitative review of psychopathology and clinical psychology research pertaining to military contexts produced from the years 1992 thought 2002 and indexed in the data base PsycINFO. There were 526 studies published in this eleven-year period. United States was by far the most productive country followed by China and United Kingdom. Military Medicine was considerably the journal that published the most studies, followed by Psychiatric Services, Journal of Clinical Psychology, and Military Psychology. Almost one-third of the papers were prevention or treatment studies.

Key words: Military psychology, psychopathology, clinical psychology, bibliometric analysis.

\section{INTRODUCCIÓN}

El ámbito militar siempre ha constituido un campo de particular importancia para la psicología, tanto la clínica como la no clínica. De hecho, la aplicación de la psicología en los contextos militares ha supuesto importantes avances en la psicopatología y la psicología clínica, a la vez que estas disciplinas han proporcio-

Correspondencia: Ana Magaz, Universidad Nacional de Educación a Distancia, Facultad de Psicología, Juan del Rosal 10 (Ciudad Universitaria), 28040 Madrid. Correo-e: anamagaz@terra.es

Parte de este trabajo fue presentado al IV Congreso de la Asociación Española de Psicología Clínica y Psicopatología, celebrado en Sevilla del 25 al 27 de marzo de 2004. nado ayuda y soporte psicológico a las personas que viven $\mathrm{y} / \mathrm{o}$ trabajan en dichos contextos (p.ej., situaciones bélicas, misiones humanitarias en otros países, etc.).

Actualmente, los campos de confluencia entre la psicología y el contexto militar son diversos. Por ejemplo, Gal y Mangelsdorff (1991), en su libro Handbook of military Psychology identificaron seis líneas de investigación y actuación de la psicología en los contextos militares. La primera se refería a la selección de personal y a su adecuación al puesto. En relación con esta línea han destacado históricamente los trabajos de Robert $M$. Yerkes y sus compañeros de la División 
de Psicología del Ejército de Estados Unidos (EEUU), que en el transcurso de la I Guerra Mundial desarrollaron los Tests Alpha y Beta de Inteligencia General (véase Anastasi, 1968). Estos test fueron utilizados para identificar el nivel de inteligencia de los reclutas, para tomar decisiones administrativas sobre la asignación de puestos y para la admisión de oficiales instructores. Esta tradición continuó durante la II Guerra Mundial. Así por ejemplo, el Ejército estadounidense desarrolló en 1939 una nueva prueba, el Test de Clasificación General del Ejército, que se aplicó a más de 12 millones de hombres. A partir de ese momento las pruebas psicométricas adquirieron especial importancia para la selección y clasificación de personal en general (Johnson, 1991).

La segunda línea de investigación se centra en analizar los efectos que sobre el rendimiento militar tienen distintos factores humanos. La investigación se ha centrado sobre dos aspectos del estrés, el primero de ellos hace referencia a los diferentes activadores del estrés que están presentes en las situaciones de combate y sus efectos sobre el rendimiento, y el segundo a las estrategias que han demostrado ser más efectivas para reducir y controlar el estrés en las poblaciones militares (Bartone, 1998). La tercera línea de investigación ha examinado la influencia de distintos factores medioambientales sobre la actuación militar. Así, se estudia el rendimiento humano en ambientes muy cálidos, muy fríos o, muy ruidosos, mientras se actúa en altitudes muy altas, en presencia de gases tóxicos, trabajando en vehículos de combate que alcanzan altas tasas de aceleración y vibración, o sistemas armamentísticos que conllevan enfermedades del sistema motriz, con el fin de mejorar el rendimiento de los combatientes en el campo de batalla. La cuarta línea de investigación se ha referido a la capaci- dad de mando en la actuación militar. Antes de la II Guerra Mundial la investigación sobre liderazgo se centraba en constructos relacionados con características innatas como inteligencia y rasgos de personalidad. Las cuatro décadas posteriores a esta guerra están marcadas por amplias investigaciones empíricas sobre el liderazgo cara a cara, y es en los últimos años cuando se produce la fusión entre las teorías organizacionales y las del liderazgo, considerando que el constructo liderazgo debe incluir la influencia indirecta al igual que la tradicional directa cara a cara, así como conceptos sobre cultura organizacional, poder, política de organización, teoría de sistemas y estrategias de dirección. La quinta línea de investigación hace referencia a la conducta individual y de grupo, en concreto a la moral que es definida como una función de cohesión y espíritu de cuerpo. Son estos términos los que van a fundamentar muchas de las investigaciones realizadas en los últimos años. Y por último, la sexta línea de investigación se centra en la intervención clínica y consultiva, con tareas de evaluación, prevención y tratamiento de los soldados y sus familiares durante los extensos períodos de paz y apoyo a las fuerzas combatientes durante los períodos de conflicto (Nichols, 1991). Adicionalmente, en los manuales al uso sobre psicología militar suelen abordarse ciertos contenidos especiales como guerra psicológica, acciones psicológicas, información y desinformación, persuasión, técnicas propagandísticas, negociación en el rescate de rehenes, medidas contra el terrorismo, y aspectos psicológicos relacionados con la rehabilitación y adaptación de prisioneros de guerra.

Como puede apreciarse, a partir de las seis líneas básicas trazadas por Gal y Mangelsdorff (1991) se observa que la mayor parte de ellas incluyen contenidos relacionados con la psicopatología, la 
psicología clínica y la psicología de personalidad. Así por ejemplo, la primera línea incluye contenidos de psicopatología, personalidad, evaluación y diagnóstico, entre otros. Las líneas dos y tres se centran fundamentalmente en contenidos de psicopatología (especialmente la segunda; p.ej., efectos de las situaciones de estrés militar sobre el desarrollo de problemas y trastornos mentales). La línea 5, aparte de los contenidos propios de la psicología social, también hace referencia a contenidos de psicopatología y psicología de la personalidad (p.ej., estrés laboral, satisfacción laboral). Finalmente, en relación con la línea sexta cabría decir que se trata de un ámbito en el que predominan estrictamente los contenidos de psicología clínica (evaluación, prevención y tratamiento).

El estudio de estos aspectos psicológicos relacionados con el contexto militar ha adquirido recientemente un especial interés en nuestro país tras la reciente profesionalización de las Fuerzas Armadas. Actualmente es elevado el número de soldados profesionales que permanecen en el Ejército durante periodos prolongados de tiempo, en los diferentes puestos y en relación con distintas funciones (de formación, bélicas, humanitarias, etc.), lo cual justifica una mayor atención psicológica a este tipo de profesionales.

Mediante el presente trabajo hemos intentado llevar a cabo una primera aproximación al estudio de los aspectos psicopatológicos y clínicos relacionados con los profesionales del ámbito militar. Para ello, nuestro objetivo se ha centrado en la realización de un análisis bibliométrico sobre la literatura científica relacionada con la psicopatología y psicología clínica desarrolladas en el contexto militar. En nuestro análisis hemos seguido una orientación similar a las llevadas a cabo por otros autores que han efectuado estudios objetivos sobre el estado de la litera- tura científica centrada en cuestiones clínicas (p.ej., Báez et al., 1996; Quiles et al., 2000).

Con objeto de centrar nuestro estudio, nos hemos limitado al periodo correspondiente a la última década (1992 al 2002). Por otra parte, y puesto que el campo de la psicología militar excede los propósitos de la presente investigación, en nuestro análisis nos hemos centrado exclusivamente en contenidos relacionados con satisfacción laboral, personalidad, psicopatología, prevención y tratamiento de los trastornos psicológicos. Estos cambos están directamente implicados en el bienestar y salud de los soldados profesionales, así como también en su ajuste y desempeño de sus labores profesionales. En nuestros análisis nos hemos basado tanto en el análisis de la productividad (anual y por países y autores) como en el análisis por materias (revistas más productivas, tipo de publicación, y análisis temático de los trabajos) (véase Quiles et al., 2000).

\section{MÉTODO}

El análisis bibliométrico se ha llevado a cabo exclusivamente a partir de la base de datos PsycINFO (periodo 1992-2002, ambos inclusive). Esta base de datos contiene más de un millón de citas y resúmenes de artículos de revistas, capítulos de libros, conferencias e informes técnicos, todos referidos al campo de la psicología. El fondo documental de revistas incluye material internacional seleccionado de más de 1.700 publicaciones periódicas, en más de 35 idiomas. También recoge información sobre temas psicológicos tratados en otras disciplinas como medicina, psiquiatría, sociología, educación, farmacia, antropología, fisiología, lingüística y derecho. PsycINFO es sin duda la base de datos que incluye la información más exhaustiva sobre los 
tópicos del presente estudio; se descartaron otras posibles bases de datos (p.ej., Medline o Current Contents) por considerar que la información que podrían aportar sería mayoritariamente redundante o de escasa relevancia para la presente investigación.

Una vez establecida la base de datos fuente, se procedió a diseñar la estrategia de búsqueda que permitiera seleccionar aquellos documentos que estuvieran relacionados con los campos establecidos. Para ello se definieron las siguientes variables de búsqueda: personalidad, psicopatología, prevención y tratamiento de trastornos psicológicos, y satisfacción laboral; todo ello en relación con el contexto militar. Los descriptores utilizados fueron: (1) "personalit " and militar" " para la variable personalidad, (2) "psychol* disorder and militar" " y "psychopathol ${ }^{*}$ and militar ${ }^{*}$ " para la variable psicopatología, (3) «mental health prevention and militar " $\mathrm{y}$ "mental health treatment and militar" " para la variable prevención y tratamiento de trastornos psicológicos, y (4) «job satisfaction and militar » para la variable satisfacción laboral. El rango de edad utilizado fue a partir de los 18 años (i.e., 18 años o más), edad a partir de la cual se puede ingresar en las Fuerzas Armadas.

Una vez cotejados todos los trabajos para cerciorarnos de que no se contabilizaban los mismos documentos dos veces $\mathrm{y}$, eliminados aquellos que trataban exclusivamente sobre aspectos biológicos o médicos, se creó una base de datos de trabajo con los documentos obtenidos. El total de trabajos que componen la base bibliográfica sobre la cual se realizó el análisis bibliométrico fue de 526 , de los cuales, $162(30,8 \%)$ están vinculados al campo temático personalidad, 143 $(27,2 \%)$ a psicopatología, $169(32,1 \%)$ a prevención y tratamiento de trastornos psicológicos, y $52(9,9 \%)$ a satisfacción laboral.

\section{RESULTADOS}

En el análisis de los datos y en la presentación de los mismos seguiremos un planteamiento general similar al efectuado recientemente por Quiles et al. (2000). Presentaremos en primer lugar los datos sobre el análisis de productividad (anual, por países, y por autores) y posteriormente los relacionados con el análisis de materias (tipos de publicación, productividad por revistas, y análisis temático).

\section{Análisis de la productividad}

Los resultados relativos a la producción anual se indican en la Tabla 1. En la tabla presentamos la distribución anual de los trabajos seleccionados durante el período 1992-2002 para cada una de las variables estudiadas y también de forma conjunta. Tal y como puede observarse en esta tabla, los años de mayor productividad total fueron 1996 y 2002, y el año donde la productividad total fue menor fue 1992. Para la variable personalidad, el año de mayor productividad fue 1996 y los de menor productividad 1992 y 1998. En la variable psicopatología, el año de mayor productividad fue 1996 y el de menor productividad 1999. Para la variable prevención y tratamiento de trastornos psicológicos, el año de mayor productividad fue 2002 y los de menor productividad 1993 y 1994. Y finalmente, para la variable satisfacción laboral, los años más productivos fueron $2001 \mathrm{y}$ 2002, y el menos productivo 1999. Puede observarse, así mismo, que 1996 es el año más productivo tanto de trabajos sobre psicopatología (27 trabajos) como sobre personalidad (22 trabajos). Se observa un decremento con el tiempo de estudios centrados en estas dos variables, mientras que se produce un incremento en las variables más aplicadas (prevención y tratamiento, y satisfacción laboral). 
Tabla 1. Productividad anual (número de trabajos) en los cuatro campos temáticos (personalidad, psicopatología, prevención y tratamiento de trastornos psicológicos, y satisfacción laboral), así como la productividad total (período 1992-2002)

\begin{tabular}{lccccc}
\hline & $\begin{array}{c}\text { Personalidad } \\
(N=162)\end{array}$ & $\begin{array}{c}\text { Psicopatología } \\
(N=143)\end{array}$ & $\begin{array}{c}\text { Prevención } y \\
\text { tratamiento } \\
(N=169)\end{array}$ & $\begin{array}{c}\text { Satisfacción laboral } \\
(N=52)\end{array}$ & $\begin{array}{c}\text { Total } \\
(N=526)\end{array}$ \\
\hline 1992 & 9 & 11 & 8 & 3 & 31 \\
1993 & 10 & 19 & 7 & 4 & 40 \\
1994 & 17 & 10 & 7 & 3 & 37 \\
1995 & 17 & 10 & 14 & 4 & 45 \\
1996 & 22 & 27 & 10 & 3 & 62 \\
1997 & 11 & 9 & 22 & 4 & 46 \\
1998 & 9 & 15 & 18 & 6 & 48 \\
1999 & 17 & 7 & 16 & 2 & 42 \\
2000 & 14 & 18 & 18 & 6 & 56 \\
2001 & 20 & 9 & 12 & 9 & 50 \\
2002 & 16 & 8 & 37 & 8 & 69 \\
\hline
\end{tabular}

Tabla 2. Productividad (número de trabajos) de los 25 países con al menos un trabajo, según los 4 campos temáticos

\begin{tabular}{|c|c|c|c|c|c|}
\hline & $\begin{array}{c}\text { Personalidad } \\
(N=162)\end{array}$ & $\begin{array}{l}\text { Psicopatología } \\
(N=143)\end{array}$ & $\begin{array}{c}\text { Prevención y } \\
\text { tratamiento } \\
(N=169)\end{array}$ & $\begin{array}{c}\text { Satisfacción } \\
\text { labonal } \\
(N=52)\end{array}$ & $\begin{array}{c}\text { Total } \\
(N=526)\end{array}$ \\
\hline EEUU & 128 & 113 & 138 & 45 & 424 \\
\hline China & 7 & 3 & 11 & - & 21 \\
\hline Reino Unido & $\mathbf{9}$ & 4 & $\mathbf{5}$ & 1 & 19 \\
\hline Alemania & 2 & 4 & 5 & - & 11 \\
\hline Holanda & 2 & 2 & 3 & 1 & 8 \\
\hline España & 1 & 2 & - & 1 & 4 \\
\hline Canadá & 1 & 1 & - & 2 & 4 \\
\hline Italia & 1 & 2 & 1 & - & 4 \\
\hline Australia & - & 1 & 2 & 1 & 4 \\
\hline Israel & - & 2 & 1 & - & 3 \\
\hline India & - & 2 & - & 1 & 3 \\
\hline Turquía & 1 & 2 & - & - & 3 \\
\hline Rusia & 1 & 2 & - & - & 3 \\
\hline Suiza & - & 2 & - & - & 2 \\
\hline Polonia & 1 & 1 & - & - & 2 \\
\hline Francia & 1 & - & 1 & - & 2 \\
\hline Repub. de Eslovenia & 1 & - & - & - & 1 \\
\hline Países Escandinavos & 1 & - & - & - & 1 \\
\hline México & 1 & - & - & - & 1 \\
\hline Bélgica & 1 & - & - & - & 1 \\
\hline Rumania & 1 & - & - & - & 1 \\
\hline Japón & 1 & - & - & - & 1 \\
\hline Pakistán & 1 & - & - & - & 1 \\
\hline Brasil & - & - & 1 & - & 1 \\
\hline Nueva Zelanda & - & - & 1 & - & 1 \\
\hline
\end{tabular}


Tabla 3. Productividad por autores (frecuencia y porcentaje) según la clasificación propuesta por Crane (1969)

\begin{tabular}{lccccc}
\hline & $\begin{array}{c}\text { Personalidad } \\
(N=302)\end{array}$ & $\begin{array}{c}\text { Psicopatología } \\
(N=339)\end{array}$ & $\begin{array}{c}\text { Prevención y } \\
\text { tratamiento } \\
(N=298)\end{array}$ & $\begin{array}{c}\text { Satisfacción } \\
\text { laboral } \\
(N=92)\end{array}$ & $\begin{array}{c}\text { Total } \\
(N=823)\end{array}$ \\
\hline Grandes productores & - & $1(0,29)$ & $1(0,34)$ & - & $2(0,24)$ \\
Productores moderados & $2(0,66)$ & $2(0,58)$ & $4(1,34)$ & - & $11(1,33)$ \\
Aspirantes & $27(8,94)$ & $27(7,96)$ & $25(8,41)$ & $2(2,17)$ & $121(14,72)$ \\
Transeúntes & $273(90,40)$ & $310(91,46)$ & $267(89,89)$ & $90(97,83)$ & $689(83,71)$ \\
\hline
\end{tabular}

Nota. "En la productividad total los autores sólo se contabilizaron una vez aunque aparecieran en más de un campo temático.

En relación con la productividad por países (véase la Tabla 2), podemos observar que, teniendo en cuenta los cuatro campos de aplicación, el análisis de los 25 países incluidos (con al menos un trabajo) refleja que la mayor parte de los trabajos corresponden a E.U., lugar donde se efectuó el $80,6 \%$ de toda la productividad. El resto, corresponde en su mayoría a estudios pertenecientes a cuatro países (China, Reino Unido, Alemania y Holanda). España se encuentra igualada en estudios a Canadá, Italia y Australia (con cuatro trabajos). El resto de los países (16 en total) sólo han producido entre 3 y 1 trabajo. Cuando este análisis se realiza para cada una de las cuatro variables de estudio, consideradas de forma independiente, se obtienen resultados similares a los expuestos anteriormente, siendo E.U., China, Reino Unido, Alemania y Holanda los países más productivos para cada una de ellas (excepto para satisfacción laboral).

Finalmente, se analizó la productividad por autores, cuyos datos se indican en la Tabla 3. En los 526 trabajos recogidos intervienen un total de 823 autores lo que supone una media de 1,56 autores por trabajo. En dicha tabla presentamos la distribución de los autores según su número de firmas para el total de la muestra y para cada una de las variables objeto de estudio consideradas de forma independiente. Para realizar esta distribución seguimos la clasificación pro- puesta por Crane (1969; Quiles et al., 2000) quien establece cuatro categorías de productividad: grandes productores (con 10 o más trabajos), productores moderados (entre 5 y 9 trabajos), aspirantes (entre 2 y 4 trabajos) y transeúntes (1 trabajo). Como puede apreciarse en la Tabla 3, autores con 10 o más trabajos (grandes productores) únicamente aparecen en las variables prevención y tratamiento de los trastornos psicológicos y psicopatología; en la variable satisfacción laboral sólo se registran autores con menos de 5 trabajos (i.e., aspirantes y transeúntes). En la Tabla 4 se especifican los autores más productivos para el total de la muestra de trabajos. Se trata de una distribución piramidal muy apuntada con una base muy amplia formada por autores transeúntes que aportan una sola firma (689 autores, $83,71 \%$ del total), mientras que en el otro extremo, los autores más prolíficos, constituyen un grupo muy reducido (sólo 2 autores han publicado más de 10 artículos, $0,24 \%$ del total). Los autores con más trabajos publicados son Robert Rosenheck para la variable prevención y tratamiento de trastornos psicológicos, y Christopher Fruech para la variable psicopatología.

\section{Análisis por materias}

En primer lugar se efectuó el análisis según el tipo de publicación. La base de 
Tabla 4. Autores más productivos $(\mathrm{N}=823)$ : Número de trabajos en los que aparece cada autor, agrupados según la clasificación de Crane (1969)

\begin{tabular}{|c|c|c|c|}
\hline \multicolumn{4}{|c|}{ Grandes Productores } \\
\hline Robert Rosenheck & \multicolumn{3}{|l|}{35} \\
\hline B. Christopher Fruech & \multicolumn{3}{|l|}{12} \\
\hline \multicolumn{4}{|c|}{ Productores Moderados } \\
\hline Lee Hyer & 7 & Michael Neale & 6 \\
\hline Rani A. Hoff & 7 & Michael Windle & 6 \\
\hline Benjamin G. Druss & 6 & J. William Albrecht & $\mathbf{5}$ \\
\hline Alan Fontana & 6 & Jon D. Elhai & $\mathbf{5}$ \\
\hline W. Brad Johnson & 6 & Rakesh Lall & $\mathbf{5}$ \\
\hline Richard P. Marshall & 6 & & \\
\hline \multicolumn{4}{|c|}{ Aspirantes } \\
\hline Brett t. Litz & 4 & Linda k. Hervig & 3 \\
\hline Paul T. Bartone & 4 & Raymond E. King & 3 \\
\hline Kerry Chamberlain & 4 & Thomas Koehler & 3 \\
\hline Paul B. Gold & 4 & Nigel Long & 3 \\
\hline David T. Orman & 4 & Carol Macdonald & 3 \\
\hline Frederick M. Siem & 4 & Patrick H. Munley & 3 \\
\hline Ross R. Vickers & 4 & Rainer Richter & 3 \\
\hline Antoni Florkowski & 3 & Leonora Rosen & 3 \\
\hline James Reich & 3 & M. David Rudd & 3 \\
\hline Erig G. Carbone & 3 & Gro M. Sandal & 3 \\
\hline Jeffrey Cigrang & 3 & John A. Schinka & 3 \\
\hline Matthew Dobson & 3 & Daniel W. Smith & 3 \\
\hline Fritz Drasgow & 3 & Marilyn Stolar & 3 \\
\hline David Forbes & 3 & F. Suzanne Talbert & 3 \\
\hline Matthew Friedman & 3 & Frank W. Weathers & 3 \\
\hline David A. Gryson & 3 & 90 Autores & 2 \\
\hline \multicolumn{4}{|c|}{ Transeúntes } \\
\hline 689 Autores & 1 & & \\
\hline
\end{tabular}

datos PsycINFO incluye citaciones y resúmenes sobre cuatro tipos de publicaciones: artículos de revistas, capítulos de libros, conferencias, e informes técnicos. En la Tabla 5 presentamos la distribución de los tipos de publicación para cada una de las variables de análisis y para el total de la muestra. Como puede apreciarse en dicha tabla, el artículo de revista es el tipo de publicación que más aparece (representa el $82,3 \%$ del total de las publicaciones recogidas), en todos y cada uno de los cuatro campos estudiados. El tipo de publicación que menos aparece para cada una de las variables examinadas y para el total de la muestra es el informe técnico $(1,1 \%)$.
Respecto a la productividad por revistas, el análisis realizado muestra que de los 526 trabajos localizados, 433 $(82,3 \%)$ pertenecía a revistas científicas (162 revistas en total). En la Tabla 6 aparecen las 21 revistas más productivas (con 4 o más artículos publicados), indicándose el número y porcentaje de artículos publicados en cada uno de los cuatro campos objeto de estudio, así como de forma conjunta. La revista más productiva para cada una de las variables estudiadas de forma independiente y para el total de la muestra fue Military Medicine, con un total de 73 artículos, que representan el núcleo de 
Tabla 5. Tipo de publicación (artícula, conferencia, capítulo de libro, informe) en función del campo temático

\begin{tabular}{lccccc}
\hline & $\begin{array}{c}\text { Personalidad } \\
(N=162)\end{array}$ & $\begin{array}{c}\text { Psicopatología } \\
(N=143)\end{array}$ & $\begin{array}{c}\text { Prevención } y \\
\text { tratamiento } \\
(N=169)\end{array}$ & $\begin{array}{c}\text { Satisfacción } \\
\text { laboral } \\
(N=52)\end{array}$ & $\begin{array}{c}\text { Total } \\
(N=526)\end{array}$ \\
\hline Artículo & 131 & 131 & 143 & 28 & 433 \\
Conferencia & 22 & 8 & 18 & 23 & 71 \\
Capítulo & 5 & 3 & 8 & - & 16 \\
Informe & 4 & 1 & - & 1 & 6 \\
\hline
\end{tabular}

Tabla 6. Productividad de las revistas (entre paréntesis se indican los porcentajes relativos a cada categoría temática y al total)

\begin{tabular}{|c|c|c|c|c|c|}
\hline & $\begin{array}{l}\text { Personalidad } \\
(N=131)\end{array}$ & $\begin{array}{l}\text { Psicopatología } \\
\qquad(N=131)\end{array}$ & $\begin{array}{c}\text { Prevención y } \\
\text { tratamiento } \\
(N=143)\end{array}$ & $\begin{array}{c}\text { Satisfacción } \\
\text { laboral } \\
(N=28)\end{array}$ & $\begin{array}{c}\text { Total } \\
(N=433)\end{array}$ \\
\hline Military Medicine & $15(11,45)$ & $10(7,63)$ & $42(29,37)$ & $6(21,42)$ & $73(16,85)$ \\
\hline Psychiatric Services & - & $5(3,81)$ & $15(10,48)$ & - & $20(4,61)$ \\
\hline Journal of Clinical Psychology & $9(6,87)$ & $7(5,34)$ & - & - & $16(3,69)$ \\
\hline Military Psychology & $13(9,92)$ & - & - & $3(10,71)$ & $16(3,69)$ \\
\hline Journal of Personality Assessment & $11(8,39)$ & $3(2,29)$ & - & - & $14(3,23)$ \\
\hline Chinese Mental Health Journal & $4(3,05)$ & $3(2,29)$ & $7(4,89)$ & - & $14(3,23)$ \\
\hline Journal of Traumatic Stress & $2(1,52)$ & $8(6,10)$ & $3(2,09)$ & - & $13(3,00)$ \\
\hline Journal of Nervous \& Mental Disease & - & $7(5,34)$ & $2(1,39)$ & - & $9(2,07)$ \\
\hline American Journal of Psychiatry & - & $5(3,81)$ & $4(2,79)$ & - & $9(2,07)$ \\
\hline Medical Care & - & - & $8(5,59)$ & - & $8(1,84)$ \\
\hline Psychological Reports & $3(2,29)$ & $5(3,81)$ & - & - & $8(1,84)$ \\
\hline Personality \& Individual Differences & $4(3,05)$ & $3(2,29)$ & - & - & $7(1,61)$ \\
\hline Assessment & $5(3,81)$ & $1(0,76)$ & - & - & $6(1,38)$ \\
\hline Psychological Assessment & $4(3,05)$ & $2(1,52)$ & - & - & $6(1,38)$ \\
\hline Psychologische Beitrage (Alemania) & - & $2(1,52)$ & $4(2,79)$ & - & $6(1,38)$ \\
\hline Chinese Journal of Clinical Psychology & $2(1,52)$ & - & $3(2,09)$ & - & $5(1,15)$ \\
\hline Hospital \& Community Psychiatry & - & $2(1,52)$ & $3(2,09)$ & - & $5(1,15)$ \\
\hline Journal of Career Assessment & - & $1(0,76)$ & - & $3(10,71)$ & $4(0,92)$ \\
\hline Journal of Applied Psychology & $3(2,29)$ & - & - & $1(3,57)$ & $4(0,92)$ \\
\hline Journal of Substance Abuse Treatment & - & $4(3,05)$ & - & 一 & $4(0,92)$ \\
\hline Journal of Abnormal Psychology & $1(0,76)$ & $3(2,29)$ & - & - & $4(0,92)$ \\
\hline \multicolumn{5}{|c|}{11 revistas publicaron 3 artículos cada una de ellas } & $33(7,62)$ \\
\hline \multicolumn{5}{|c|}{24 revistas publicaron 2 artículos cada una de ellas } & $48(11,08)$ \\
\hline \multicolumn{5}{|c|}{106 revistas publicaron 1 artículo cada una de ellas } & $106(23,45)$ \\
\hline
\end{tabular}

las publicaciones específicas de este ámbito. Esta revista, fundada en 1891, es la revista oficial de la Asociación de Cirujanos Militares de E.U. Su objetivo es ofrecer un foro de discusión sobre los últimos avances científicos en medicina militar, así como de otros problemas relacionados con el cuidado y mantenimiento de la salud en los profesionales militares.
Finalmente, en relación con el análisis temático se observa que el mayor número de trabajos (casi un tercio) corresponde al campo temático de prevención y tratamiento (169 trabajos $(32,1 \%)$, seguido de personalidad (162 trabajos, 30,8\%) y psicopatología (143 trabajos, $27,2 \%$ ), y a mayor distancia se encuentra satisfacción laboral (52 trabajos, 9,9\%). Para los campos de personalidad y psicopatología 
se efectuó un análisis de contenidos, encontrándose que en personalidad se daba una equiparación entre los trabajos relativos a rasgos y procesos de personalidad y los relacionados con la evaluación de variables de personalidad (i.e., $50 \%$ de trabajos en cada uno de los dos subcampos). En cambio, en psicopatología se detectaron 75 trabajos $(52,4 \%)$ relacionados con abuso de sustancias, 50 trabajos (35\%) relacionados con neurosis y trastornos de personalidad, y 18 trabajos $(12,6 \%)$ se asociaban a otros trastornos psicológicos.

\section{DISCUSIÓN Y CONCLUSIONES}

La finalidad de la presente investigación se ha centrado en examinar las publicaciones científicas sobre la psicopatología y psicología clínica relacionadas con el ámbito militar partiendo de un análisis bibliométrico centrado en los 4 campos temáticos siguientes: personalidad, psicopatología, prevención y tratamiento de los trastornos psicológicos, y satisfacción laboral, y partiendo de la base de datos PsycINFO (periodo 1992-2002). Seleccionamos estas cuatro variables por su relación con la salud y el bienestar de los profesionales militares, así como con la adaptación y el desempeño en su contexto profesional. El número total de trabajos seleccionados fue de 526, de los cuales 162 se correspondían con la variable personalidad, 143 con la variable psicopatología, 169 con la variable prevención y tratamiento de trastornos psicológicos, y 52 con la satisfacción laboral.

La evolución sobre la producción de trabajos sobre psicopatología y psicología clínica militar es irregular (tanto en lo que concierne al curso temporal como a la evolución en los diferentes campos temáticos) existiendo dos puntos claros de inflexión. El primero de ellos se produce en 1996, momento en que se incre- menta la producción en las variables de personalidad y psicopatología así como en la producción total, debido a la aparición de trabajos sobre la Guerra de los Balcanes que finaliza en 1995. El segundo punto de inflexión ocurre en el año 2002 , cuando se incrementa la producción en la variable prevención y tratamiento de trastornos psicológicos así como la producción total; el porcentaje más alto de los trabajos publicados durante este año se centran en las consecuencias del atentado terrorista en E.U. del 11 de septiembre de 2001 y en la Guerra de Afganistán (Operación Libertad Duradera). Llama la atención que durante 2002 no se haya producido un incremento en publicaciones sobre contenidos de psicopatología similar al que se da para los contenidos sobre prevención y tratamiento.

El año de menor producción es 1992; este dato difiere en algún modo con el razonamiento aquí expuesto, que hace referencia al incremento de las publicaciones tras un importante conflicto bélico, ya que en el año 1991 finaliza la Guerra del Golfo Pérsico (Operación Tormenta del Desierto). Igualmente ocurre con el hecho de que los años 1993 y 1994 sean los de menor productividad para la variable prevención y tratamiento de trastornos psicológicos, ya que en 1992 los veteranos de la Guerra del Golfo comenzaron a notificar diversos síntomas como cansancio, dolores de cabeza y articulaciones, erupciones cutáneas, diarreas, insomnio, dificultades para la concentración y fluctuaciones en el ánimo. Se estima que hasta unos 160.000 de los casi 700.000 hombres y mujeres que actuaron en la operación Tormenta del Desierto podrían sufrir estos síntomas.

El análisis de la productividad por países para las cuatro variables objeto de estudio consideradas de forma conjunta revela que EEUU es el principal país productor, explicando el $80,6 \%$ de las 
publicaciones, seguido a gran distancia por un grupo de doce países que explicarían el $16,5 \%$ de los trabajos seleccionados. Este segundo grupo esta formado por China, Reino Unido, Alemania, Holanda, España, Canadá, Italia, Australia, Israel, India, Turquía y Rusia (ordenados de mayor a menor número de artículos publicados). Y finalmente, existe un tercer grupo de doce países que explicarían el 2,8\% de los trabajos. Estos países son Suiza, Polonia, Francia, Países Escandinavos, República de Eslovenia, México, Bélgica, Rumania, Japón, Pakistán, Brasil y Nueva Zelanda. Cuando este análisis se realiza para cada una de las variables de estudio, consideradas de forma independiente, se obtienen resultados similares a los expuestos anteriormente.

Como cabría esperar, el análisis centrado en el tipo de publicación indica que el artículo de revista es el tipo de publicación que más aparece en este análisis bibliométrico, representando el $82,3 \%$ del total de las publicaciones recogidas; también es el tipo de publicación que más se registra para cada una de las variables objeto de estudio cuando son analizadas de forma independiente. El tipo de publicación que menos aparece es el informe técnico $(1,1 \%)$, también en cada una de las variables examinadas. Esto indica que la investigación psicológica en el ámbito militar sigue un patrón similar al que ocurre en otros campos de la psicología, donde los artículos de revistas especializadas suelen representar la mayor parte de la producción científica.

Uno de los aspectos que llaman la atención de los datos obtenidos en el presente estudio es la escasa representatividad de la investigación psicológica referida a la psicopatología y psicología clínica en el ámbito militar. Como puede apreciarse, la mayor parte de los artículos publicados corresponden a la revista Military Medicine, lo que sugiere que la investigación sobre psicopatología y psicología clínica militar está realizada fundamentalmente por profesionales de la medicina, más que por profesionales de la psicología. También llama la atención que sean tan escasos en este campo los grandes productores y/o productores moderados. Esto viene a indicar que aún no está suficientemente establecida el área de investigación sobre la psicopatología y psicología clínica militar, incluso si partimos de la evidencia procedente de la investigación en E.U. (en este país, aunque se ha producido un desarrollo importante, parece estar circunscrito al campo de la medicina).

Es posible que nuestro análisis no refleje suficientemente las tendencias de investigación en el campo psicológico, ya que probablemente muchos trabajos con participantes militares sobre temas de psicopatología o intervención psicológica sean publicados en revistas especializadas de psicología sin la inclusión de descriptores militares (ni en las palabras clave, ni en el título, ni en el resumen del trabajo). Esto puede darse con muchos trabajos que se publican, por ejemplo, sobre adicciones, estrés postraumático y otros trastornos asociados al estrés agudo, y explicaría que no aparezcan muchas revistas de gran prestigio en psicopatología y psicología clínica, o que otras (p.ej., Journal of Abnormal Psychology, Journal of Traumatic Stress) sólo reflejen unos pocos artículos sobre el tema. De cualquier modo, el importante desarrollo que ha experimentado la psicopatología y la psicología clínica (p.ej., Ballester, 2002; Becoña, 1999; Echeburúa, 1998; Sandín, 2002; Tobón et al., 2004) garantiza y justifica la necesidad de una mayor implicación de estas disciplinas en el contexto del militar profesional, $y$ en concreto en nuestro país. 


\section{REFERENCIAS BIBLIOGRÁFICAS}

Anastasi, A. (1968). Psychological Testing. Toronto: Macmillan.

Báez, C., Borda, M., Salaberría, K., y Echeburúa, E. (1996). El juego patológico: Un análisis bibliométrico a partir del Psychological Abstracts (1974-1994). Clínica y Salud, 7, 123-135.

Ballester, R. (2002). Adhesión terapéutica: Revisión histórica y estado de la cuestión en la infección por VIH/SIDA. Revista de Psicopatología y Psicología Clínica, 7 , 151-176.

Bartone, P. (1998). Stress in the military setting En C. Cronin (Ed.), Military psychology: An introduction (pp. 113-146). New York: Simon \& Schuster.

Becoña, E. (1999). La discrepancia entre la investigación y la práctica clínica de la terapia de conducta. Revista de Psicopatología y Psicología Clínica, 4, 71-104.

Crane, D. (1969). Social structures in a group of scientist: A test of the 'Invisible College' hypothesis. American Sociological Review, 34, 335-352.

Echeburúa, E. (1998). ¿Qué terapias psicologicas son eficaces? Un reto ante el año
2000. Revista de Psicopatología y Psicología Clínica, 3, 149-160.

Gal, R., y Mangelsdorff, A.D. (Eds.) (1991). Handbook of military psychology. New York: Wiley \& Sons.

Johnson, E.M. (1991). Foreword. En R. Gal y A.D. Mangelsdorff (Eds.), Handbook of military psychology (pp. XXI-XXIV). New York: Wiley \& Sons,

Nichols, R.S. (1991). Introduction to section 6: Clinical and consultative/organizational psychology. Handbook of military psychology (pp. 575-578). New York: Wiley \& Sons.

Quiles, M.J., Ortigosa, J.M., Pedroche, S., y Méndez, X. (2000). Investigaciones psicológicas sobre el cuidado del niño hospitalizado: Un análisis bibliométrico. Medicina Psicosomática y Psiquiatria de Enlace, 53, 27-42.

Sandín, B. (2002). Papel de las emociones negativas en el trastorno cardiovascular. Revista de Psicopatología y Psicología Clínica, 7, 1-18.

Tobón, S., Vinaccia, S., y Sandin, B. (2004). Modelo procesual del estrés en la dispepsia funcional: Implicaciones para la evaluación y el tratamiento. Revista de Psicopatología y Psicología Clínica, 9, 81-98. 